\title{
Application Value of Bedside Ultrasound in the Positioning of PICC Tips in Preterm Infants
}

\author{
Yuting Jiao ${ }^{1}$, Yumei $\mathrm{Wu}^{2}$, and Zhi Yang ${ }^{1 *}$ \\ ${ }^{1}$ Yuting Jiao, Zhi Yang, Department of Ultrasound, Binzhou Medical University Hospital, Binzhou, 256600.China \\ ${ }^{2}$ Yumei Wu, Department of NICU, Binzhou Medical University Hospital, Binzhou, 256600.China
}

\begin{abstract}
Objective To investigate the application value of bedside ultrasound in the localization of the tip of a Peripherally Inserted Central Catheter (PICC) in preterm infants. Methods 52 preterm infants underwent bedside ultrasound and bedside X-ray examination. Observing the position of the catheter tip, and using the bedside X-ray positioning as the gold standard. Statistics of the incidence of PICC tip normal position and ectopic position showed by bedside ultrasound, and comparing the difference between the results of bedside ultrasound and bedside X-ray. Calculating the accuracy, sensitivity, and specificity of ultrasound in diagnosing the tip position, and the length of the inlet and withdrawal tubes of the ectopic catheter was observed and calculated by ultrasound and down-regulated to the appropriate position under ultrasound guidance. Results The display rate of catheter tip by bedside color Doppler ultrasound was $98.0 \%$, and the accuracy rate of tip position was $90.2 \%$. There was no significant difference compared with bedside X-ray $(P=0.375)$, and the tip position of the two examination methods was highly consistent (Kappa $=0.769, P<0.001)$. The sensitivity of diagnosing tip ectopy was $76.5 \%$ and the specificity was $97.1 \%$, and the success rate of ectopic catheters in ultrasound-guided downsetting was $100 \%$. Conclusion Bedside ultrasound accurately show the position of the catheter tip and guide the entry and withdrawal of ectopic catheter, with high sensitivity and specificity, which has high value of clinical promotion.
\end{abstract}

\section{INTRODUCTION}

Early parenteral nutrition support is the key to improve the survival rate of preterm infants with imperfect development of various organs. Peripherally Inserted Central Catheter (PICC) technology is a technology that solves the difficulty of peripheral venous puncture, infusion of parenteral nutrition and stimulating drugs, and opens up venous access for the diagnosis and treatment of premature infants ${ }^{[1]}$. At this stage, clinical bedside X-rays are mainly used to locate the tip of the catheter, but it have radiation damage to children. Frequent movement of children increases the risk of infection and catheter displacement ${ }^{[2]}$. The bedside ultrasound has the advantages of no radiation damage and no need to move the child frequently. It can also guide the adjustment of the ectopic catheter in real time, judge the maintenance of the catheter, and reduce the incidence of complications ${ }^{[3]}$. The purpose of this study was to explore the accuracy of tip positioning of PICC in premature infants and its application value in catheter maintenance.

\section{MATERIALS AND METHODS}

\subsection{Study population}

A total of 52 preterm infants who underwent PICC in the neonatal intensive care unit of our hospital from 6/2019 to $5 / 2020$ were selected. The infants were catheterized through the expensive vein, median vein, axillary vein, great saphenous vein, and small saphenous vein., and all of them met the indications of PICC catheterization. Grouping: 52 children underwent bedside X-ray and bedside ultrasound examinations, including 24 boys and 28 girls, gestational age 28 to 34 weeks, average (31.75 \pm 1.84 ) weeks, body weight $0.85 \mathrm{~kg}$ to $2.45 \mathrm{~kg}$, average $(1.62 \pm 0.84) \mathrm{kg}$; the age at the time of catheterization was (1 3) days, average $(0.88 \pm 0.72)$ days. All family members of premature infants included in the study signed written informed consent.

\footnotetext{
*Corresponding author: Zhi Yang, Email: 13061030808@163.com
} 


\subsection{Instrument and methods}

\subsubsection{Instrument and data acquisition}

Mindray's M9 Expert portable ultrasound diagnostic instrument was used for bedside ultrasound. The probe models were L12-4s, L14-6Ns, and the frequency was 6.6-13MHz. The bedside X-ray radiography machine was YJM035R-A mobile high-frequency X-ray machine with A radiation volume of $0.02 \mathrm{mSV}$.

\subsubsection{Method}

After the catheter is inserted, X-ray examination is performed at the bedside and the position of the catheter tip is located. Do bedside ultrasound examination within 24 hours. Upper limb venous catheterization: The probe is placed in the 2-3 intercostal space next to the right side of the sternum to scan the long axis of the superior vena cava and the innominate vein; the probe is deflected to the left to show the right atrium entrance of the superior vena cava, look for the tip of the catheter;.lower limb venous catheterization:the probe is placed under the xiphoid process to scan the long axis of the inferior vena cava and the right atrium entrance of the inferior vena cava. The entrance of the right atrium of the lower vena cava is used as a positioning mark, and the distance between the tip of the catheter and the entrance of the right atrium of the vena cava is measured. Calculate the accuracy of the catheter tip position of the bedside ultrasound.

\subsubsection{Observation indicators}

The ultrasonographic manifestations of the PICC tip: Upper extremity venipuncture: The tip of the catheter is located in the lower $1 / 3$ of the superior vena cava or the right atrium junction of the superior vena cava; Trans-lower extremity venipuncture: the tip of the catheter is located in the inferior vena cava, above the diaphragm ${ }^{[4]}$. X-ray findings with normal PICC tip position: upper extremity venous puncture: the tip of the catheter is located in the 4th to 6th thoracic vertebrae; trans-lower limb venous puncture: the tip of the catheter is located in the 8 th to 10 th thoracic vertebra ${ }^{[2,5,6]}$. The incidence of normal and ectopic PICC tip position displayed by bedside ultrasound was counted, and compare the results of bedside ultrasound and bedside $\mathrm{X}$-ray.

For catheters that are misaligned or ectopic into the right atrium, the catheter is advanced and retracted under ultrasound guidance. For the catheter ectopic into the internal jugular vein or the contralateral subclavian vein, withdraw the catheter to the confluence of the innominate vein and the superior vena cava under ultrasound guidance (Fig. 1). The child takes a sitting position, puts the probe on the right side of the sternal longitudinal section, and guides the tip of the catheter enter the superior vena cava. After adjusting the tube, perform X-ray inspection of the tip position.

\subsection{Statistical method}

For all analyses, SPSS Statistics, version 25.0 was used. The count data was expressed as a number and rate (\%), and the measurement data was expressed as $(\bar{x} \pm s)$. The McNemar test was used to compare the difference between bedside ultrasound and bedside X-ray ectopic. The level of significance was set at $p<0.05$. Kappa test was performed on the results of the two inspection methods. A Kappa value $<0.4$ was considered as poor consistency, 0.4-0.75 was considered as moderate consistency, and $\geqslant 0.75$ was considered as excellent consistency.

\section{RESULT}

\subsection{Bedside X-ray and bedside color Doppler ultrasound tip positioning results}

A total of 52 preterm infants with PICC catheter tip were scanned by bedside X-ray. The catheter tip position was normal in 35 cases (65.4\%) and ectopic in 17 cases (34.6\%). Ultrasound results: 52 premature infants, the superior, inferior vena cava and right atrium entrance could be clearly displayed $(100 \%)$. The catheter tip was clearly displayed in 51 cases $(98.0 \%)$, and the catheter tip could not be clearly displayed in 1 case $(2.0 \%)$ due to lungs, among which 37 cases $(71.1 \%)$ had normal position. The catheter tip was ectopic in 14 cases $(26.9 \%)$ (Table 1).

\subsection{Comparison of the results of the tip positioning between bedside $X$-ray and bedside ultrasound}

Comparing 51 cases of bedside X-ray and bedside ultrasound catheter tip position normal and ectopic incidence, the coincidence rate of bedside ultrasound catheter tip position was $90.2 \%$ (47 cases), The difference between the two examination results was not statistically significant ( $P=0.375$ ), The Kappa test result showed that the two results were highly consistent $(K a p p a=0.769)$. The sensitivity and the specificity of bedside ultrasound in diagnosing the PICC tip position was calculated to be $76.5 \%$ and $97.1 \%$ (Table 2).

\subsection{The success rate of the adjustment under ultrasound guidance}

The 14 ectopic catheter tips were all adjusted to the appropriate position under ultrasound guidance, and then examined by bedside X-rays. The success rate of the adjustment was $100 \%$.

Table1. Comparison of PICC tip position in X-ray and ultrasound

\begin{tabular}{|c|c|c|c|}
\hline \multicolumn{2}{|c|}{ X-ray } & \multicolumn{2}{|c|}{ ultrasound } \\
\hline tip position & $\mathrm{n}$ & tip position & $\mathrm{n}$ \\
\hline
\end{tabular}




\begin{tabular}{cccc}
\hline C2 C6 & 4 & IJV & 4 \\
T1 & 2 & SCV & 2 \\
T3 & 3 & INV & 2 \\
T4 T6 & 29 & SVC $\sim$ RA & 31 \\
T7 T8 & 8 & RA & 6 \\
T8 T10 & 6 & IVC & 6 \\
Undetermined & 0 & Undetermined & 1 \\
Total & 52 & Total & 52 \\
\hline
\end{tabular}

$\mathrm{C}$, cervical vertebra; $\mathrm{T}$, thoracic vertebra; IJV, internal jugular vein (ipsilateral); SCV, subclavian vein (contralateral); INV, innominate vein (ipsilateral); SVC, superior vena cava; RA, right atrium; IVC, Inferior vena cava

\section{DISCUSSION}

With the increase of advanced maternal age in recent years, the birth rate of premature infants increases accordingly, and improving the survival rate of premature infants is an important issue facing neonatal department $^{[5]}$. PICC technique has become an important measure to rescue premature infants in Neonatal intensive care unit (NICU). Ectopic insertion of the catheter tip into the right atrium can cause arrhythmias, tamponema and other serious complications ${ }^{[3]}$, while too shallow catheter location will increase the incidence of thrombosis and phlebitis.Especially in premature infants, the blood vessels are relatively thin, and it is more likely to cause serious consequences if improper operation or improper catheter position occurs in the process of catheterization. Therefore, it is very important to ensure the normal position of the catheter tip during PICC catheterization in premature infants.

At present, bedside chest X-ray is still the main clinical method for PICC tip positioning, and bedsidechest $\mathrm{X}$-ray examination is still the "gold standard" for tip positioning ${ }^{[6]}$. Chest $\mathrm{X}$ - rays can only indirectly indicatethe tip position by projecting the catheter tip onto the surface of the anterior, posterior ribs, or thoracic vertebrae. In neonates, especially

Table 2 The result of bedside ultrasound judging that the tip position is normal and ectopic (\%)

\begin{tabular}{|c|c|c|c|c|c|c|c|c|}
\hline & \multicolumn{2}{|c|}{ X-ray（n) } & \multirow{2}{*}{$\begin{array}{l}\text { Total } \\
(\mathrm{n})\end{array}$} & \multirow[b]{2}{*}{ Sensitivity } & \multirow[b]{2}{*}{ Specificity } & \multirow{2}{*}{$\begin{array}{l}\text { Positive } \\
\text { predictive } \\
\text { value }\end{array}$} & \multirow{2}{*}{$\begin{array}{l}\text { Negative } \\
\text { predictive } \\
\text { value }\end{array}$} & \multirow[b]{2}{*}{ Accuracy } \\
\hline & normal & ectopic & & & & & & \\
\hline \multirow{2}{*}{ ultrasound (n) } & 33 & 4 & 37 & \multirow{3}{*}{76.5} & \multirow{3}{*}{97.1} & \multirow{3}{*}{92.9} & \multirow{3}{*}{89.2} & \multirow{3}{*}{90.2} \\
\hline & 1 & 13 & 14 & & & & & \\
\hline Total & 34 & 17 & 51 & & & & & \\
\hline
\end{tabular}
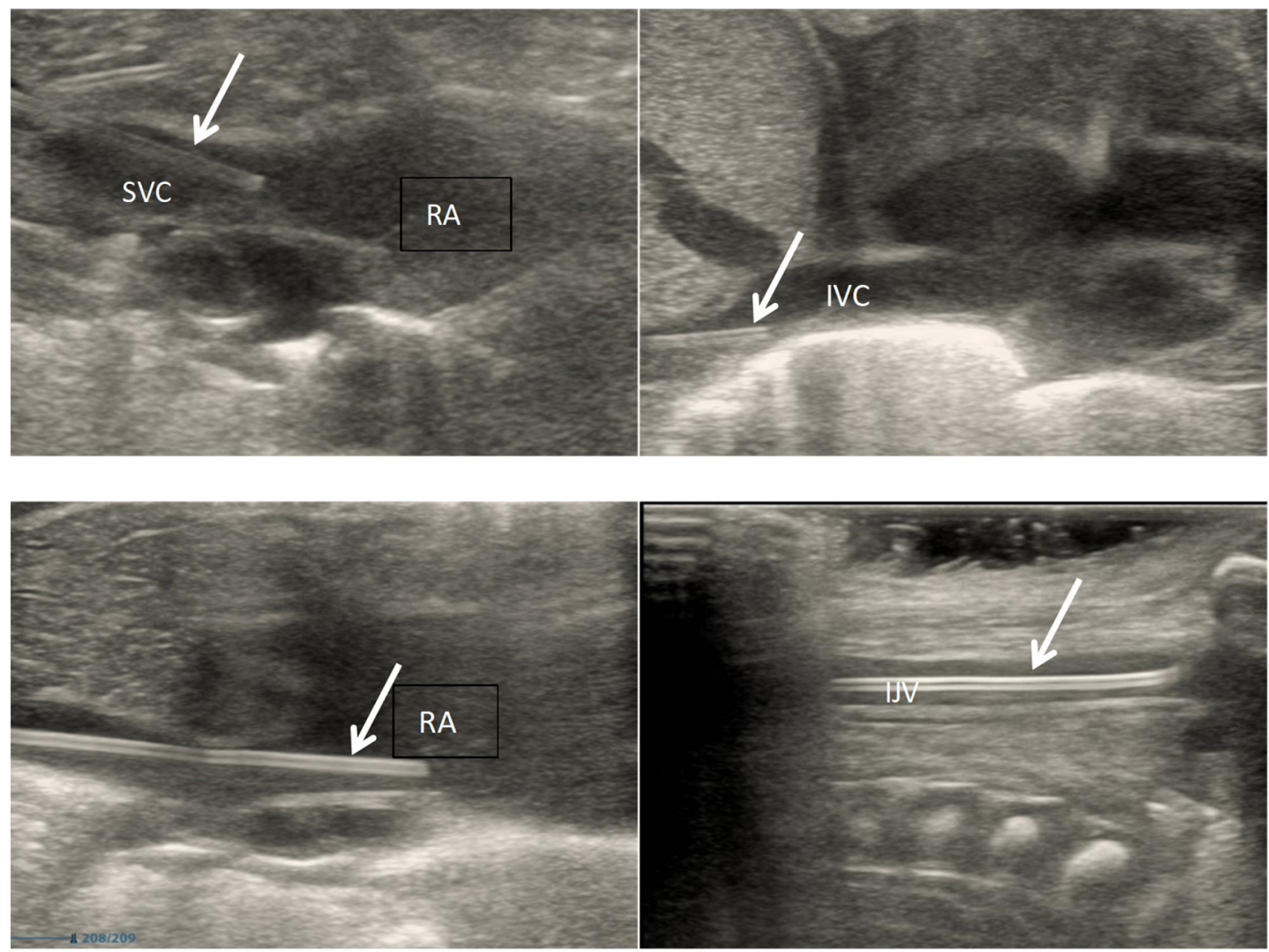

Fig.1. PICC tip position is normal and ectopic (Long arrow, PICC tube; IJV, internal jugular vein; SVC, superior vena cava; IVC, inferior vena cava; RA, right atrium ) 
premature infants with incomplete ossification of ribs. The high-frequency ultrasound probe can penetrate the costal cartilage, and the non-degenerated thymus gland can be used as the acoustic window to clearly show the superior and inferior vena cava and catheter tip. The catheter is made of silica gel or polyurethane, and the longitudinal cut of the catheter on ultrasound shows an "equal sign" echo of high and low. In the study of Ohki $\mathrm{Y}$ et al. ${ }^{[7]}$, ultrasound was consistent with the catheter tip shown by X-ray in $87 \%$ of cases, and the sensitivity of ultrasound was $78 \%$. In this study, the accuracy and sensitivity of ultrasound to determine the ectopic location of catheter tip were $90.2 \%$ and $76.5 \%$ among the results of bedside color ultrasound in 52 children.In 2 cases, bedside ultrasound showed that the tip was located at the right atrial entrance, and bedside X-ray showed that the tip was located at chest level 8. Analyses the reason of its low sensitivity may be with different will lead to the location of the catheter tip position difference, ultrasonic scan process, children with arm adduction is outreach catheter position deeper, if when placing tube catheter at the entrance right atrium, the tip of the catheter after PICC tube with children with limb movement into the right atrium, therefore, proposed to tip catheter at the entrance of right atrium TuiGuan to $0.5 \sim 2 \mathrm{~cm}$ above the entrance.

In PICC catheterization, the incidence of ectopic tip was high. Catheterization was carried out through upper limb veins, and the tip was usually located in bilateral internal jugular vein, contralateral subclavian vein, bilateral innamental vein and right atrium.Catheters were inserted through the veins of the lower extremities, and the tips were usually located at the common iliac, internal iliac and external iliac veins. Due to the lack of timeliness, bedside X-ray only temporarily locate the position of the catheter and cannot provide real-time clinical guidance for entering and returning the catheter. However, bedside ultrasound provides real-time guidance for regulating the ectopic catheter to reduce $\mathrm{X}$-ray radiation damage and reduce movement of children.In this study, 14 cases of PICC catheter were ectopic, and the catheters were all located in the normal position under ultrasound guidance.Curt et al ${ }^{[8]}$ found that, inserting the catheter under ultrasound guidance will significantly shorten the operation time and reduce the repeated friction and damage of the catheter to the vessel wall, indicating that bedside ultrasound is more advantageous than bedside X-rays in the application of real-time observation and maintenance of the catheter.

In neonatal PICC tip positioning, bedside ultrasound has limitations. First of all, it cannot display the entire catheter. In addition, due to the thin subcutaneous fat layer, ribs or lung gas in premature infants, the catheter tip of some infants is not clearly displayed.It is recommended to use a combination of cross-sectional and longitudinal scanning to find the tip of the catheter. After seeing the "equal-sign-like" echo,.Cross-sectional scan. If the intravenous dot-like hyperechoic disappears suddenly, it is the tip of the catheter.Michel et al. ${ }^{[2]}$ found that injecting saline into the catheter helped the catheter tip to show that the flow of water appeared as a hyperechoic bubble on ultrasound. Since most of the children in this hospital have upper extremity venous catheterization, and the observation cases are in normal positions, the treatment of the tip of the lower extremity venous catheterization will be further studied.

\section{CONCLUSION}

Bedside ultrasound has high sensitivity, specificity and accuracy for PICC tip positioning. It can guide the introduction and withdrawal of the ectopic catheter in real time, avoiding the radiation damage of premature infants by multiple X-rays. Therefore, it has high clinical promotion value.

\section{References}

1. ZhanZhan L;LiZhang C.Comparison of ultrasound guided modified Seldinger technique versus blind puncture for peripherally inserted central catheter: a meta-analysis of randomized controlled trials.Crit Care. [J].Critical Care,2015(19):64-65.[J].Critical Care, 2015, 19(1):64-65

2. Michel F, Brevaut-Malaty V, Pasquali R, et al.Comparison of ultrasound and X-ray in determining the position of umbilical venous catheters[J].Resuscitation, 2012, 83(6):705-713

3. Nichols I, Humphrey JP.The efficacy of upper arm placement of peripherally insertedcentral catheters usingbedside ultrasound and microintroducer technique.[J].J Infus Nurs, 2008, 31(3):165-176

4. Darling J C, Newell S J, Mohamdee O, et al.Central venous catheter tip in the right atrium: a risk factor for neonatal cardiac tamponade[J].Journal of Perinatology, 2002, 21(7):461-464

5. Xiaotian Z, Fengying Z.Study on the application value of ultrasound catheter tip positioning technology in catheter placement in premature infants[J].Chinese Journal of Ultrasound in Medicine, 2018, 34(1):40-43

6. Xiao W,Xiuhong W, Xiuxia $\mathrm{Z}$, et al. Reliable imaging markers for the optimal location of the tip of the central venous catheter inserted through the periphery[J].Chongqing Medicine,2019,48(3):525-528

7. Ohki $\mathrm{Y}$, Tabata $\mathrm{M}$, Kuwashima $\mathrm{M}$, et al.Ultrasonographic detection of very thin percutaneous central venous catheter in neonates[J].Acta paediatrica, 2000, 89(11):1381-1384

8. Curt D, Mark R, Eli S, et al.Ultrasound-guided central venous catheter placement decreases complications and decreases placement attempts compared with the landmark technique in patients in a pediatric intensive care unit[J].Critical Care Medicine, 2009, 37(3):1090-1096 